Pacific Journal of Mathematics

THE STRICT TOPOLOGY, SEPARABLE MEASURES, AND 


\title{
THE STRICT TOPOLOGY, SEPARABLE MEASURES, AND PARACOMPACTNESS
}

\author{
ROBERT F. WHEELER
}

\begin{abstract}
The theory of the strict topology $\beta$ on $C^{*}(X)$ has recently been extended to a completely regular setting by Sentilles. Here it is shown that equality of the separable and $\tau$-additive Baire measures on $X$ is a sufficient condition for $\left(C^{*}(X), \beta\right)$ to be a strong Mackey space. As a consequence, the Conway-LeCam Theorem for paracompact spaces is extended to the completely regular case. A locally convex topology $\beta_{e}$ on $C^{*}(X)$ is considered; $\beta_{e}$ is strong Mackey, and the dual space is the space of separable measures. Results of Dudley, Granirer, and Leger and Soury on convergence in the space of measures are unified and extended in this context.
\end{abstract}

The topology of a completely regular Hausdorff space $X$ can be recovered from the family of its continuous metric images. In this paper an analogous idea is developed: that theorems about convergence of functions and measures on metric spaces can be used (via an inductive limit procedure) to obtain results in a completely regular setting. The principal consequences are the determination of a sufficient condition for equality of the Mackey and strict topologies on $C^{*}(X)$, and a unification and extension of known results on convergence in the space of Baire measures on $X$.

The concepts introduced here arise from the theory of the strict topology for completely regular spaces due to Dennis Sentilles [17]. He considers locally convex topologies $\beta_{0}, \beta$, and $\beta_{1}$ on $C^{*}(X)$ which extend the strict topology of Buck [2] in the locally compact setting and also relate to the theory of measures on topological spaces developed in, for example, Varadarajan [18]. In this paper the emphasis is on continuous maps from $X$ to metric spaces $Y$ and the induced maps on $\left(C^{*}(Y), \beta\right)$ to $C^{*}(X)$. This leads to introduction of an inductive limit topology $\beta_{e}$ on $C^{*}(X)$, intermediate between $\beta$ and $\beta_{1}$.

The topology $\beta_{e}$ has a number of significant properties. In particular, $\left(C^{*}(X), \beta_{e}\right)$ is always a strong Mackey space. Using the notion of partition of unity, it is then shown that $\beta=\beta_{e}$ when $X$ is paracompact; hence the Conway-LeCam Theorem $[4,12]$ extends to the completely regular setting. Moreover, the dual space of $\left(C^{*}(X), \beta_{e}\right)$ can be represented as $M_{S}(X)$, the space of separable measures introduced by Dudley [5], or as the space of linear func- 
tionals on $C^{*}(X)$ whose restrictions to bounded equicontinuous sets are continuous in the pointwise topology, a notion considered by Leger and Soury [13]. Results of these authors and Granirer [9] on convergence in the space of measures are interpreted and extended in this context, emerging as consequences of results in the duality theory of locally convex spaces.

1. Definitions and preliminary results. All spaces $X$ are henceforth assumed to be completely regular and Hausdorff, except that pseudometrics $d$ on a space $X$ will be considered. In this case $d$-closed, $d$-separable, etc. refer to properties of the pseudometric space $(X, d)$. The space of real-valued bounded continuous functions on $X$ is denoted by $C^{*}(X)$. A partition of unity (POU) on $X$ is a subfamily $\left(f_{\alpha}\right)$ of $C^{*}(X)$ such that $0 \leqq f_{\alpha} \leqq 1 \forall \alpha, \Sigma f_{\alpha}=1$, and $\left\{x: f_{\alpha}(x) \neq 0\right\}$ is a locally finite family of subsets of $X$. A POU $\left(f_{\alpha}\right)$ is subordinate to an open cover $\left(U_{\alpha}\right)$ of $X$ if $\left\{x: f_{\alpha}(x)>0\right\} \subset U_{\alpha} \forall_{\alpha}$. There is a POU subordinate to any open cover of a paracompact space (in particular, a metric space).

Basic references for the duality theory of topological vector spaces and topological measure theory are [16] and [18], respectively. A zero-set in $X$ is a set of the form $f^{-1}(0)$, where $f \in C^{*}(X)$. A cozeroset is the complement of a zero-set. The Baire sets are the least $\sigma$ algebra containing the zero-sets, and we deal primarily with Baire measures: see [18] for the definitions of $M(X), M_{\sigma}(X), M_{\tau}(X)$, and $M_{t}(X)$. If $A \subset M(X)$, then $A^{+}=\left\{\mu^{+}: \mu \in A\right\}$, and $A^{-}=\left\{\mu^{-}: \mu \in A\right\}$. If $\mu \in M(X)$, then the support of $\mu$ is

$$
\cap\{Z: Z \text { a zero-set, }|\mu|(Z)=|\mu|(X)\} \text {. }
$$

Similarly, if $A \subset M(X)$, then the (uniform) support of $A$ is

$$
\bigcap\{Z:|\mu|(Z)=|\mu|(X) \forall \mu \in A\} \text {. }
$$

A $\tau$-additive measure on a metric space has separable support.

If $A$ is a directed set and $\left(f_{\alpha}\right)_{\alpha \in A}$ a family in $C^{*}(X)$, then $f_{\alpha} \downarrow 0$ if $\left(f_{\alpha}\right)$ converges monotonically pointwise to 0 ; similarly we have such notations as $Z_{\alpha} \downarrow \varnothing$ and $f_{\alpha} \uparrow 1$.

The symbol LCS means locally convex Hausdorff topological vector space. If $E, F$ are linear spaces in duality, then the weak and Mackey topologies on $E$ with respect to $F$ are denoted, as usual, by $\sigma(E, F)$ and $\tau(E, F)$. A subset $A$ of a topological space $X$ is relatively countably compact if every sequence in $A$ has a cluster point in $X$. We will say, following Conway [4] that a LCS is a strong Mackey space if every relatively weak*-countably compact subset of the dual space is equicontinuous. 
Topologies $\beta_{0}, \beta$, and $\beta_{1}$ on $C^{*}(X)$ which yield $M_{t}, M_{\tau}$, and $M_{v}$, respectively, as dual spaces, are introduced by Sentilles [17]; independently, Fremlin, Garling, and Haydon [7] have considered topologies $T_{t}, T_{\tau}$, and $T_{\sigma}$ which perform the same function. Happily, these topologies are pairwise equivalent (i.e., $\beta_{0}=T_{t}, \beta=T_{\tau}$, and $\beta_{1}=T_{\sigma}$ ), and we note several crucial results in this theory. A subset $A$ of $M_{\tau}$ is uniformly $\tau$-additive if, for any $f_{\alpha} \downarrow 0$ in $C^{*}(X)$ and $\varepsilon>0$, there is an index $\alpha_{0}$ such that, for $\alpha \geqq \alpha_{0},\left|\mu\left(f_{a}\right)\right|<\in \forall \mu \in A$; there is, similarly, a notion of uniform $\sigma$-additivity.

Proposition 1.1 [7, 17] (a). $\left(C^{*}(X), \beta_{1}\right)$ is a strong Mackey space, and the families of relatively weak*-countably compact and uniformly $\sigma$-additive subsets of $M_{\sigma}(X)$ coincide. Moreover, if $A \subset M_{\sigma}(X)$, then $A$ has either of these properties if and only if $A^{+}$ and $A^{-}$do. (b) If $A \subset M_{-}(X)$, then $A$ is uniformly $\tau$-additive if and only if $A^{+}$and $A^{-}$are uniformly $\tau$-additive. Also $\beta=\beta_{1}$ if and only if $M_{\tau}=M_{\sigma}$, and, in this case, $\beta$ is strong Mackey.

Any continuous map $\varphi: X \rightarrow Y$ induces a map $T: C^{*}(Y) \rightarrow C^{*}(X)$ defined by $T(f)=f \circ \varphi$. Then $T$ is $\beta_{0}-\beta_{0}, \beta-\beta$, and $\beta_{1}-\beta_{1}$ continuous [14]; the adjoint $T^{*}: M(X) \rightarrow M(Y)$ is weak ${ }^{*}$-continuous and preserves $M_{o}, M_{\tau}$, and $M_{t}$. Finally, $\mathscr{P}$ is the topology on $C^{*}(X)$ of pointwise convergence on $X$.

2. Metric spaces. The work of Fremlin, Garling, and Haydon [7] has established that $\left(C^{*}(X), \beta_{0}\right)$ is a strong Mackey space whenever $X$ is complete metric. In this case, $\beta=\beta_{0}$, and so the following theorem, whose proof relies on their basic technique, is a stronger result.

THEOREM 2.1. If $X$ is a metric space, then $\left(C^{*}(X), \beta\right)$ is a strong Mackey space.

Proof. Let $d$ be a metric for $X$, and let $A$ be a relatively weak*-countably compact subset of $M_{\tau}(X)$. We establish first that for any $\varepsilon>0$ and $\delta>0$, there is a finite subset $Y$ of $X$ such that $|\mu|(X \backslash N(Y, \delta)) \leqq \varepsilon$ (here $N(Y, \delta)=\{x \in X: d(x, Y) \leqq \delta\})$. If this fails for some particular $\varepsilon$ and $\delta$, we construct sequences of measures $\left(\mu_{n}\right)$ in $A$ and finite subsets $\left(Y_{n}\right)$ of $X$ such that
(1) $Y_{0}=\varnothing$;
(2) $\left|\mu_{n}\right|\left(X \backslash N\left(\bigcup_{i<n} Y_{i}, \delta\right)\right)>\varepsilon$;
(3) $Y_{n} \subset X \backslash N\left(\bigcup_{i<n} Y_{i}, \delta\right)$;
(4) $\left|\mu_{n}\right|\left(N\left(Y_{n}, \delta / 4\right)\right)>\varepsilon \forall n$. 
Indeed there exists $\mu_{1} \in A$ with $\left|\mu_{1}\right|(X)>\varepsilon$, and it is an immediate consequence of $\tau$-additivity, applied to the family of open $\delta / 4$-spheres, that $Y_{1}$ can be chosen so that (3) and (4) are valid. If $\mu_{i}$ and $Y_{i}$ have been chosen for $i<n$, then, by hypothesis, some $\mu_{n} \in A$ satisfies (2). Applying $\tau$-additivity of $\left|\mu_{n}\right|$ to the family of open $\delta / 4$-spheres centered at points of $X \backslash N\left(\bigcup_{i<n} Y_{i}, \delta\right)$, we obtain $Y_{n}$ satisfying (3) and (4). Thus, by induction, $\left(\mu_{n}\right)$ and $\left(Y_{n}\right)$ are completely defined.

Let $G_{n}=\left\{x: d\left(x, Y_{n}\right)<\delta / 3\right\}, H_{n}=N\left(Y_{n}, \delta / 4\right)$. Since $\left|\mu_{n}\right|\left(H_{n}\right)>\varepsilon$, it is easy to see that there is an $f_{n} \in C^{*}(X)$ with

$$
\left\|f_{n}\right\| \leqq 1, f_{n} \mid X \backslash G_{n} \equiv 0
$$

and $\left|\int f_{n} d \mu_{n}\right|>\varepsilon$. Define $T: \iota^{\infty} \rightarrow C^{*}(X)$ as follows: for each

$$
\alpha=\left(\alpha_{n}\right) \in \ell^{\infty}, T(\alpha)=\Sigma \alpha_{n} f_{n} .
$$

(that $T(\alpha)$ is continuous follows from the fact that $d\left(G_{m}, G_{n}\right) \geqq \delta / 3$ for $m \neq n)$.

Now $T$ is $\sigma\left(\ell^{\infty}, \ell^{1}\right)-\sigma\left(C^{*}, M_{\tau}\right)$ continuous (direct verification), hence the adjoint $T^{*}: M_{\tau}(X) \rightarrow \ell^{1}$ is $\sigma\left(M_{\tau}, C^{*}\right)-\sigma\left(\ell^{1}, \ell^{\infty}\right)$ continuous. Thus $T^{*}(A)$ is relatively weakly countably compact in $\ell^{1}$, and so is relatively norm compact. But if $e_{n}$ is the $n$th unit vector in $\ell^{1}$, then $\left|T^{*} \mu_{n}\left(e_{n}\right)\right|=\left|\mu_{n}\left(f_{n}\right)\right|>\varepsilon$, and this contradiction establishes the truth of the original claim.

Hence for each $m$ and $n$ there is a finite subset $F_{m, n}$ of $X$ with $|\mu|\left(X \backslash N\left(F_{m, n}, 1 / 2^{n}\right)\right) \leqq\left(m \cdot 2^{n}\right)^{-1} \forall \mu \in A$. If $S$ is the closure of

$$
\bigcup_{m=1}^{\infty} \bigcap_{n=1}^{\infty} N\left(F_{m, n}, 1 / 2^{n}\right),
$$

then $S$ is separable and $|\mu|(X \mid S)=0 \forall \mu \in A$.

For each $\mu \in A$, let $\mu_{S}$ denote the restriction to the Baire (=Borel) subsets of $S$. If $\left(\mu_{n}\right)$ is a sequence in $A$ with a weak*-cluster point $\mu_{0} \in M_{\tau}(X)$, then $\left|\mu_{0}\right|(X \mid S)=0$ [18, p. 183]. Moreover, every member of $C^{*}(S)$ extends to a member of $C^{*}(X)$, and it follows easily that $\left(\mu_{n}\right)_{S}$ clusters to $\left(\mu_{0}\right)_{S}$ in $M_{\tau}(S)$. Thus, $A_{1}=\left\{\mu_{S}: \mu \in A\right\}$ is relatively weak*-countably compact in $M_{\tau}(S)$. But $S$ is Lindelöf, hence $M_{o}(S)=$ $M_{\tau}(S)[18, \mathrm{p} .175]$, so that $A_{1}$ is $\beta$-equicontinuous and therefore uniformly $\tau$-additive in $M_{\tau}(S)$, by 1 . 1b. Thus, if $f_{\alpha} \downarrow 0$ in $C^{*}(X)$, and $g_{\alpha}=f_{\alpha} \mid S$, then for each $\varepsilon>0$ there is an index $\alpha_{0}$ such that, for $\alpha \geqq \alpha_{0},\left|\int_{X} f_{\alpha} d \mu\right|=\left|\int_{S} g_{\alpha} d \mu_{S}\right|<\varepsilon \forall \mu \in A$. Hence $A$ is uniformly $\tau$ additive, and therefore is $\beta$-equicontinuous.

COROLLARY 2.2. A relatively weak*-countably compact set of $\tau$ - 
additive measures on a metric space has a uniformly separable support.

It is well-known that $M_{*}^{*}(X)$ is weak*-metrizable if (and only if) $X$ is metrizable. This fails for $M_{\tau}(X)$ (unless $X$ is finite), but a crucial property of metric spaces, formalized in the notion of an angelic space [15], can still be recovered for the entire space of measures.

Corollary 2.3. If $X$ is a metric space, then $\left(M_{-}(X), w^{*}\right)$ is an angelic space.

Proof. According to [15], it suffices to show that if $A$ is a relatively weak*-countably compact subset of $M_{z}(X)$, then its weak*closure $\bar{A}$ is weak*-compact and consists precisely of the limits of sequences in $A$. The first assertion is clear, since $A$ is $\beta$-equicontinuous (2.1). For the second, note that $A^{+}$and $A^{-}$are uniformly $\tau$-additive (1.1b); hence they are relatively weak*-compact in $M_{*}^{+}(X)$. Let $\left(\mu_{\alpha}\right)$ be a net in $A$ with $\mu_{\alpha} \stackrel{w^{*}}{\rightarrow} \mu_{\text {. The }}$ There are measures $\mu_{1}$ and $\mu_{2}$ in $M_{*}^{+}(X)$ and a subnet $\left(\mu_{\alpha_{\beta}}\right)$ with $\mu_{\alpha_{\beta}}^{+} \stackrel{w^{*}}{\rightarrow} \mu_{1}, \mu_{\alpha_{\beta}}^{-} \stackrel{w^{*}}{\rightarrow} \mu_{2}$. Let $d$ be a metric for $\left(M_{*}^{+}(X), w^{*}\right)$ and choose $\lambda_{n}=\alpha_{\beta_{n}}$ with

$$
d\left(\mu_{\lambda_{n}}^{+}, \mu_{1}\right)<1 / n, d\left(\mu_{\lambda_{n}}^{-}, \mu_{2}\right)<1 / n .
$$

Since necessarily $\mu=\mu_{1}-\mu_{2}$, it follows that $\mu_{\lambda_{n}} \stackrel{w^{*}}{\rightarrow} \mu$. This completes the proof.

Applying [15, Theorem 0.5], if $X$ is metric, then any subspace of $M_{\tau}(X)$, endowed with any locally convex topology finer than the weak*-topology, is angelic.

3. Approximating completely regular spaces by metric spaces. If $X$ is a completely regular Hausdorff space, let $\mathscr{D}$ denote the family of all bounded continuous pseudometrics (BCPM's) on $X$. If $d \in \mathscr{D}$, then the relation: $x \sim y$ if and only if $d(x, y)=0$ is an equivalence relation on $X$, and the collection of equivalence classes $\bar{x}$ is made into a metric space $X_{d}$ by defining $\bar{d}(\bar{x}, \bar{y})=d(x, y)$. Then the natural map $\pi_{d}: X \rightarrow X_{d}$ defined by $\pi_{d}(x)=\bar{x}$ is continuous and induces maps $T_{d}: C^{*}\left(X_{d}\right) \rightarrow C^{*}(X), T_{d}^{*}: M(X) \rightarrow M\left(X_{d}\right)$.

Let $\xi$ (or $\xi(X)$, if confusion might arise) denote the family of uniformly bounded equicontinuous subsets of $C^{*}(X)$. Since arguments involving $\xi$ and partitions of unity (POU) play a prominent role in the remainder of this paper, we record a number of elementary results for purposes of reference and in an effort to standardize notation. 
Proposition 3.1 (a). If $E \in \xi(X)$, then

$$
d(x, y)=\sup \{|f(x)-f(y)|: f \in E\}
$$

is a BCPM on $X$. If $f \in E$, then $\bar{f}(\bar{x})=f(x)$ is a well-defined member of $C^{*}\left(X_{d}\right)$, and $E_{d}=\{\bar{f}: f \in E\} \in \xi\left(X_{d}\right)$. Also $T_{d}$ is a homeomorphism of $E_{d}$ onto $E$ (each being endowed with the appropriate pointwise topology), and $T_{d}(\bar{f})=f$. (b) If $\left(g_{\alpha}\right)$ is a POU on $X_{d}$, for some $d \in \mathscr{D}$, then $\left(T_{d}\left(g_{\alpha}\right)\right)$ is a POU on $X$. (c) If $\left(f_{\alpha}\right)_{\alpha \in A}$ is a POU on $X$, then $d(x, y)=\Sigma\left|f_{\alpha}(x)-f_{\alpha}(y)\right| \in \mathscr{D}$. If $\mathscr{F}=\{F \subset A: F$ finite $\}$, and, for each $F \in \mathscr{F}, \psi_{F}=\Sigma\left\{f_{\alpha}: \alpha \in F\right\}, \Gamma_{F}=\Sigma\left\{\bar{f}_{\alpha}: \alpha \in F\right\}$, then

$$
\left\{\psi_{F}: F \in \mathscr{F}\right\} \in \xi(X) \text {, }
$$

$\left(1-\psi_{F}\right) \downarrow 0$ in $C^{*}(X), \Gamma_{F} \uparrow 1$ in $C^{*}\left(X_{d}\right)$, and $T_{d}\left(\Gamma_{F}\right)=\psi_{F}$.

DeFinition 3.2. The topology $\beta_{e}$ is the finest locally convex topology on $C^{*}(X)$ such that $T_{d}:\left(C^{*}\left(X_{d}\right), \beta\right) \rightarrow C^{*}(X)$ is continuous $\forall d \in \mathscr{D}$.

The existence of such a topology, and a description of its zeroneighborhood base, are well-known [16, p. 79].

Proposition 3.3. $\beta \leqq \beta_{e} \leqq \beta_{1}$.

Proof. Each $T_{d}:\left(C^{*}\left(X_{d}\right), \beta\right) \rightarrow\left(C^{*}(X), \beta\right)$ is continuous [14], and so $\beta \leqq \beta_{e}$. Since $\beta_{1}$ is Mackey, it now suffices to show that any $\beta_{e^{-}}$ continuous linear functional $\Phi$ is a member of $M_{\sigma}$. If $\left(f_{n}\right) \in C^{*}(X)$ and $\left\|f_{n}\right\| \rightarrow 0$, then $E=\left\{f_{n}\right\}$ is a member of $\xi$ and gives rise to a BCPM $d$ (as in 3.1). Then $\Phi \circ T_{d} \in\left(C^{*}\left(X_{d}\right), \beta\right)^{\prime}=M_{\tau}\left(X_{d}\right) \subset M\left(X_{d}\right)$, so that $\Phi\left(f_{n}\right)=\left(\Phi \circ T_{d}\right)\left(\bar{f}_{n}\right) \rightarrow 0$. Thus $\Phi \in M(X)$, and a similar argument applied to $f_{n} \downarrow 0$ in $C^{*}(X)$ shows that $\Phi$ is $\sigma$-additive.

Evidently if $\Phi \in M(X)$, then $\Phi$ is $\beta_{e}$-continuous if and only if $\Phi \circ T_{d}=T_{d}^{*}(\Phi) \in M_{\tau}\left(X_{d}\right) \forall d \in \mathscr{D}$. Let $M_{S}(X)$ denote the space of $\beta_{e^{-}}$ continuous linear functionals; in $\S 4$ it will be proved that $M_{S}$ coincides with the space of separable measures introduced by Dudley [5] and the space $M X$ considered by Leger and Soury [13, p. 379].

Proposition 3.4. $\left(C^{*}(X), \beta_{e}\right)$ is a strong Mackey space.

Proof. In view of the definition of $\beta_{e}$, and 2.1, this follows immediately from standard duality arguments.

We now show (in several steps) that whenever $X$ is paracompact, 
$\beta=\beta_{e}$; hence $\beta$ is strong Mackey.

Lemma 3.5. If $\mu \in M_{o}(X)$, then the following are equivalent: (1) $\mu \in M_{S}(X)$; (2) $\mu^{+}$and $\mu^{-} \in M_{S}(X)$; (3) $|\mu| \in M_{S}(X)$.

Proof. Since $T_{d}^{*}: M_{S}(X) \rightarrow M_{\tau}\left(X_{d}\right)$ is a positive linear map, it is easy to see that (3) implies (2). Clearly (2) implies (1) and (3). Thus it suffices to show that if $\mu \in M_{S}(X)$, then $\mu^{+} \in M_{S}(X)$. Note that $\left(T_{d}^{*} \mu\right)^{+} \neq T_{d}^{*}\left(\mu^{+}\right)$in general.

Fix $d \in \mathscr{D}$, and let $\left(Z_{\alpha}\right)_{\alpha \in A}$ be a net of zero-sets in $X_{d}$ with $Z_{\alpha} \downarrow \varnothing$. Let $\left(g_{\alpha}\right)_{\alpha \in I}$ be a POU on the metric space $X_{d}$ such that $g_{\alpha} \mid Z_{\alpha} \equiv 0 \forall_{\alpha}$, and let $f_{\alpha}=T_{d}\left(g_{\alpha}\right)=g_{\alpha} \circ \pi_{d} \forall \alpha$, with $\mathscr{F}$ and $\psi_{F}$ defined as in 3.1.

Now $\mu=\mu^{+}-\mu^{-}$is the Jordan decomposition of $\mu$, and there is a Baire set $A \subset X$ such that $\mu^{+}(B)=\mu(A \cap B)$ for every Baire set $B$. Given $\varepsilon>0$, there is a zero-set $Z$ and a cozero-set $U$ with $Z \subset A \subset U$ and $|\mu|(U \backslash Z)<\varepsilon / 2$ [18, p. 164]. Choose $h \in C^{*}(X)$, with $0 \leqq h \leqq 1, h|X \backslash U \equiv 0, h| Z \equiv 1$. Then for any $\varphi \in C^{*}(X)$,

$\mu^{+}(\varphi)=\int_{A} \varphi d \mu=\int_{X} \varphi h d \mu-\int_{U \backslash Z} \varphi h d \mu+\int_{A \backslash Z} \varphi d \mu \leqq \mu(\varphi \cdot h)+\varepsilon \cdot\|\varphi\| \cdot$ In particular, if $\varphi_{F}=1-\psi_{F}$, then $\mu^{+}\left(\varphi_{F}\right) \leqq \mu\left(\varphi_{F} \cdot h\right)+\varepsilon$ 。

Now $\left\{\varphi_{F}: F \in \mathscr{F}\right\}$ is a member of $\xi$ (3.1c), hence so is

$$
\left\{\varphi_{F} \cdot h: F \in \mathscr{F}\right\} \text {. }
$$

Let $d_{1}(x, y)=\sup \left\{\left|\varphi_{F}(x) \cdot h(x)-\varphi_{F}(y) \cdot h(y)\right|: F \in \mathscr{F}\right\}$ be the corresponding member of $\mathscr{D}$, and denote the image of $x$ under $\pi_{d_{1}}$ by $\hat{x}$. Then $j_{F}(\hat{x})=\varphi_{F}(x) \cdot h(x)$ is a well-defined member of $C^{*}\left(X_{d_{1}}\right)$, and $T_{d_{1}}\left(j_{F}\right)=\varphi_{F} \cdot h$. Since $j_{F} \downarrow 0$ and $T_{d_{1}}^{*} \mu \in M_{\tau}\left(X_{d_{1}}\right), \mu\left(\varphi_{F} \cdot h\right)=T_{d_{1}}^{*} \mu\left(j_{F}\right) \rightarrow 0$. Thus for some finite subset $F_{0}$ of $H, F \supset F_{0}$ implies $\mu\left(\varphi_{F} \cdot h\right)<\varepsilon$, so that $\mu^{+}\left(\varphi_{F}\right)<2 \varepsilon$.

Let $F_{0}=\left\{\alpha_{1}, \cdots, \alpha_{n}\right\}$; then

$$
2 \varepsilon>\mu^{+}\left(1-\sum_{i=1}^{n} f_{\alpha_{i}}\right)=T_{d}^{*}\left(\mu^{+}\right)\left(1-\sum_{i=1}^{n} \bar{f}_{\alpha_{i}}\right)>T_{d}^{*}\left(\mu^{+}\right)\left(\bigcap_{i=1}^{n} Z_{\alpha_{i}}\right) .
$$

It now follows that $T_{d}^{*}\left(\mu^{+}\right)\left(Z_{\alpha}\right) \rightarrow 0$, hence $T_{d}^{*} \mu^{+} \in M_{r}\left(X_{d}\right)$, and so (since $d \in \mathscr{D}$ was arbitrary) $\mu^{+} \in M_{S}^{+}(X)$. This completes the proof.

LEMMA 3.6. The topology $\beta_{e}$ coincides with the topology of uniform convergence on weak*-compact subsets of $M_{S}^{+}(X)$.

Proof. In view of 3.4, the topology of uniform convergence on weak*-compact subsets of $M_{S}^{+}(X)$ is coarser than $\beta_{e}$. To prove the converse, it suffices to show that if $A$ is relatively weak*-countably 
compact in $M_{S}(X)$, then $A^{+}$has the same property in $M_{S}^{+}(X)$. Now $A$ is relatively weak*-countably compact in $M_{\sigma}(X)$, hence $\beta_{1}$-equicontinuous (1.1a). Thus $A^{+}$is $\beta_{1}$-equicontinuous and so if $\left(\mu_{n}\right)$ is any sequence in $A$, then $\left(\mu_{n}^{+}\right)$has a weak*-cluster point $\lambda$ in $M_{\sigma}^{+}(X)$. We show that $\lambda \in M_{S}^{+}(X)$.

Now each $\mu_{n}^{+} \in M_{S}^{+}(X)$, by 3.5 ; hence if $d \in \mathscr{D}$, then

$$
T_{d}^{*}\left(\mu_{n}^{+}\right) \in M_{\tau}^{+}\left(X_{d}\right) .
$$

Let $D_{n}$ be a closed separable subset of $X_{d}$ such that

$$
T_{d}^{*} \mu_{n}^{+}\left(X_{n} \backslash D_{n}\right)=0 \text {. }
$$

Then $S=\operatorname{cl} \bigcup_{n=1}^{\infty} D_{n}$ is separable, and $T_{d}^{*} \mu_{n}^{+}\left(X_{d} \backslash S\right)=0 \forall n$. Now $T_{d}^{*} \lambda \in M_{\sigma}^{+}(X)$ is a weak ${ }^{*}$-cluster point of $\left(T_{d}^{*} \mu_{n}^{+}\right)$, and so $T_{d}^{*} \lambda\left(X_{d} \backslash S\right)=0$.

Since $T_{d}^{*} \lambda$ is concentrated on a separable subspace of $X_{d}$, it is $\tau$-additive. Thus $\lambda \in M_{S}^{+}(X)$, completing the proof.

Proposition 3.7. If $X$ is completely regular, then the following are equivalent: (1) $\beta=\beta_{e}$; (2) $M_{\tau}(X)=M_{S}(X)$; (3) $M_{\tau}^{+}(X)=M_{S}^{+}(X)$. In this case, $\left(C^{*}(X), \beta\right)$ is a strong Mackey space.

Proof. It is shown in [17] and [18] that each of 3.5 and 3.6 remains true if $\beta_{e}$ is replaced by $\beta$ and $M_{S}$ is replaced by $M_{\tau}$. The proof now follows immediately.

Now we can establish the promised generalization of the ConwayLeCam Theorem.

Proposition 3.8. If $X$ is paracompact, then $\left(C^{*}(X), \beta\right)$ is a strong Mackey space.

Proof. Let $\mu \in M_{S}^{+}(X)$. If $Z_{\alpha} \downarrow \varnothing$, let $U_{\alpha}=X \backslash Z_{\alpha}$, and let $\left(f_{\alpha}\right)$ be a POU subordinate to $\left(U_{\alpha}\right)$. Define $d,\left(\bar{f}_{\alpha}\right)$, and $\left(\Gamma_{F}\right)$ as in 3.1(c); then $T_{d}^{*} \mu \in M_{\tau}\left(X_{d}\right)$ and $\left(1-\Gamma_{F}\right) \downarrow 0$ in $C^{*}\left(X_{d}\right)$, so $T_{d}^{*} \mu\left(1-\Gamma_{F}\right) \rightarrow 0$. Given $\varepsilon>0$, choose $F_{0}=\left\{\alpha_{1}, \cdots, \alpha_{n}\right\}$ such that

$$
\mu\left(1-\Sigma\left\{f_{\alpha}: \alpha \in F_{0}\right\}\right)=T_{d}^{*} \mu\left(1-\Gamma_{F_{0}}\right)<\varepsilon .
$$

It follows that $\mu\left(\bigcap_{i=1}^{n} Z_{\alpha_{i}}\right)<\varepsilon$, and so $\mu \in M_{\tau}^{+}(X)$. An application of 3.7 completes the proof.

COROLlary 3.9. If $X$ is paracompact, then every relatively weak*-countably compact subset of $M_{\tau}(X)$ has uniformly Lindelöf support.

Proof. If $A$ is relatively weak*-countably compact in $M_{z}(X)$, 
then $A$ is uniformly $\tau$-additive, hence so are $A^{+}$and $A^{-}$. Thus we may as well assume that $A \subset M_{\tau}^{+}(X)$. Let

$$
C=\bigcap\{Z: \mu Z=\mu X \forall \mu \in A\},
$$

and let $\left(U_{\alpha}\right)_{\alpha \in A}$ be an open cover of $C$. For each $\alpha$ choose an open subset $V_{\alpha}$ of $X$ with $V_{\alpha} \cap C=U_{\alpha}$, and let $V_{\alpha_{0}}=X \backslash C, A_{1}=A \cup\left\{\alpha_{0}\right\}$. Then $\left(V_{\alpha}\right)_{\alpha \in A_{1}}$ is an open cover of $X$; choose a POU $\left(f_{\alpha}\right)_{\alpha \in A_{1}}$ subordinate to it, and define $d$ and $\left(\bar{f}_{\alpha}\right)_{\alpha \in A_{1}}$ as in 3.1(c).

Now $T_{d}^{*} A$ is relatively weak*-countably compact in $M_{\tau}^{+}\left(X_{d}\right)$ and so there is a closed separable subset $B$ of $X_{d}$ such that $\mu\left(X \backslash \pi_{d}^{-1} B\right)=$ $T_{d}^{*} \mu\left(X_{d} \backslash B\right)=0 \forall \mu \in A$ (2.2). Since $\pi_{d}^{-1} B$ is a zero-set in $X$, we have $C \subset \pi_{d}^{-1} B$ by definition of $C$. Let $\left(x_{n}\right)$ be a sequence in $C$ such that $\left(\bar{x}_{n}\right)$ is dense in $\pi_{d}(C)$, and let $A_{2}=\left\{\alpha \in A_{1}: f_{\alpha}\left(x_{n}\right)>0\right.$ for some $\left.n\right\}$. Then $A_{2}$ is countable, and $\alpha_{0} \notin A_{2}$. If $p \in C$ and $f_{\alpha}(p)=0 \forall \alpha \in A_{2}$, then $\bar{d}\left(\bar{p}, \bar{x}_{n}\right)=d\left(p, x_{n}\right) \geqq \sum_{\alpha \in A_{2}}\left|f_{\alpha}(p)-f_{\alpha}\left(x_{n}\right)\right|=1 \forall n$, contradicting denseness of $\left(\bar{x}_{n}\right)$. Thus $C \subset \bigcup\left\{V_{\alpha}: \alpha \in A_{2}\right\}$, and so $C=\bigcup\left\{U_{\alpha}: \alpha \in A_{2}\right\}$. This completes the proof.

4. Characterizations of $\beta_{e}$ and its dual. In order to relate $\beta_{e}$ and $M_{S}$ to the work of Dudley [5], Granirer [9], and Leger and Soury [13], we obtain some alternate descriptions of these spaces.

Proposition 4.1. If $\mu \in M_{\sigma}(X)$, then the following are equivalent: (1) $\mu \in M_{S}(X)$; (2) [5] For each BCPM $d$ on $X$, there is a d-separable $d$-zero set $Z_{d}$ with $|\mu|\left(X \backslash Z_{d}\right)=0$; (3) [13] The restriction of $\mu$ to each member of $\xi$ is $\mathscr{P}$-continuous; (4) If $\left(f_{\alpha}\right)$ is equicontinuous and $f_{\alpha} \downarrow 0$, then $\mu\left(f_{\alpha}\right) \rightarrow 0$.

Proof. (1) $\rightarrow$ (2): If $d \in \mathscr{D}$, then $T_{d}^{*}(|\mu|) \in M_{\tau}\left(X_{d}\right)$, from 3.5 . Hence there is a closed separable subset $D$ of $X_{d}$ with

$$
T_{d}^{*}(|\mu|)\left(X_{d} \backslash D\right)=0,
$$

and $Z_{d}=\pi_{d}^{-1} D$ is the desired set. $(2) \rightarrow(1)$ : For any fixed $d, \pi_{d}\left(Z_{d}\right)$ is a closed separable subset of $X_{d}$ on which $T_{d}^{*}(|\mu|)$ is concentrated; thus $T_{d}^{*}(|\mu|) \in M_{\tau}\left(X_{d}\right)$ and so $|\mu| \in M_{S}(X)$. Now apply 3.5. (1) $\rightarrow(3)$ : Let $E \in \xi$, and let $\left(f_{\alpha}\right)$ be a net in $E$ which is pointwise convergent to $f_{0} \in E$. We may assume that $E$ is convex and circled. Let $g_{\alpha}=$ $\left(f_{\alpha}-f_{0}\right) / 2$, and let $h_{\alpha}(x)=\sup \left\{\left|g_{\lambda}(x)\right|: \lambda \geqq \alpha\right\}$. Applying the equicontinuity of $E$, we have each $h_{\alpha}$ continuous, $\left\{h_{\alpha}\right\} \in \xi$, and $h_{\alpha} \downarrow 0$. Let $d(x, y)=\sup \left\{\left|h_{\alpha}(x)-h_{\alpha}(y)\right|\right\}$, and use 3.1(a) to form $\left(\varphi_{\alpha}\right)$ in $C^{*}\left(X_{d}\right)$ with $T_{d}\left(\varphi_{\alpha}\right)=h_{\alpha}$ and $\varphi_{\alpha} \downarrow 0$. Then since $T_{d}^{*}(|\mu|) \in M_{\tau}\left(X_{d}\right)$,

$$
\mu \mid\left(h_{\alpha}\right)=\left(T_{d}^{*}|\mu|\right)\left(\varphi_{\alpha}\right) \rightarrow 0 \text {. }
$$


But $\left|\mu\left(g_{\alpha}\right)\right| \leqq|\mu|\left(h_{\alpha}\right)$, and so $\mu\left(f_{\alpha}\right) \rightarrow \mu\left(f_{0}\right)$. (3) $\rightarrow(4)$ : Obvious. $(4) \rightarrow(1)$ : First we show that $\mu^{+}$and $\mu^{-}$have the stated property. Indeed, for any $\varepsilon>0$, we can use the argument of 3.5 to find $h \in C^{*}(X), 0 \leqq h \leqq 1$, with $\mu^{+}(f) \leqq \mu(f \cdot h)+\varepsilon \cdot\|f\| \forall f \in C^{*}(X)$. If $\left(f_{\alpha}\right)$ is equicontinuous and $f_{\alpha} \downarrow 0$, then $\left(f_{\alpha} \cdot h\right)$ has the same properties. Fixing $\alpha_{0}$, we have $\mu^{+}\left(f_{\alpha}\right) \leqq \mu\left(f_{\alpha} \cdot h\right)+\varepsilon \cdot\left\|f_{\alpha_{0}}\right\|$ for $\alpha \geqq \alpha_{0}$, and it follows that $\mu^{+}\left(f_{\alpha}\right) \rightarrow 0$. Thus we may assume that $\mu$ is positive. If $d \in \mathscr{D}$ and $Z_{\alpha} \downarrow \varnothing$ in $X_{d}$, let $\left(g_{\alpha}\right)$ be a POU on $X_{d}$ subordinate to the cover $\left(X \backslash Z_{\alpha}\right)$. Then $\left(f_{\alpha}\right)=\left(T_{d} g_{\alpha}\right)$ is a POU on $X(3.1(\mathrm{~b}))$, and the equicontinuous net $\left(1-\psi_{F}\right)$ (notation as in 3.1) satisfies $\left(1-\psi_{F}\right) \downarrow 0$. Thus $\mu\left(1-\psi_{F}\right) \rightarrow 0$, and it follows that $T_{d}^{*} \mu \in M_{\tau}\left(X_{d}\right)$.

We note that the classes (2), (3), and (4) must share the property of (1) which is expressed by 3.5 .

Proposition 4.2. The topology $\beta_{e}$ is the finest locally convex topology on $C^{*}(X)$ which coincides with the pointwise topology $\mathscr{P}$ when restricted to uniformly bounded equicontinuous sets.

Proof. The existence of a topology $\mathscr{T}$ satisfying condition of the theorem, and a description of its zero-neighborhood base, are well-known [3, 8]. To show that $\beta_{e} \leqq \mathscr{T}$, it suffices (by 3.6) to show that if $A$ is a weak*-compact subset of $M_{S}^{+}(X), E \in \xi$, and $\left(f_{\alpha}\right)$ is a net in $E$ which is pointwise convergent to $f \in E$, then $\mu\left(f_{\alpha}\right) \rightarrow \mu(f)$ uniformly with respect to $\mu \in A$. Since, for each $d \in \mathscr{D}, T_{d}^{*} A$ is weak*-compact and therefore uniformly $\tau$-additive in $M_{\tau}^{+}\left(X_{d}\right)$ (by 2.1), the result follows from simple modifications of the proof that $(1) \rightarrow(3)$ in 4.1 .

Conversely, if $\mu$ is a $\mathscr{T}$-continuous linear functional on $C^{*}(X)$, then the restriction of $\mu$ to each member of $\xi$ is $\mathscr{P}$-continuous. It follows, upon considering sequences $\left(f_{n}\right)$ with $\left\|f_{n}\right\| \rightarrow 0$ or $f_{n} \downarrow 0$, that $\mu \in M_{\sigma}(X)$; hence $\mu \in M_{S}(X)$, by 4.1. Since $\beta_{e}$ is Mackey, we must have $\mathscr{T} \leqq \beta_{e}$. Thus $\mathscr{T}$ and $\beta_{e}$ coincide.

Corollary 4.3(a). If $X$ is paracompact, then $\beta$ is the finest locally convex topology which coincides with $\mathscr{P}$ on uniformly bounded equicontinuous sets; (b) If $X$ is paracompact locally compact or complete metric, then $\beta_{0}$ has the same property.

Proof. If $X$ is paracompact, then $\beta=\beta_{e}$ was proved in 3.7 and 3.8. The spaces mentioned in (b) satisfy $\beta_{0}=\beta$.

If $X$ is locally compact, then the $\beta$-totally bounded, relatively $\beta$-compact, and uniformly bounded equicontinuous subsets of $C^{*}(X)$ coincide. Thus the first part of (b) was essentially obtained by Collins 
and Dorroh [3, Theorem 6.4], and now emerges as bearing an unanticipated relation to the Mackey problem.

Let $\tau(\xi)$ denote the topology on $M(X)$ of uniform convergence on members of $\xi$. The second part of the next theorem is proved in [13], but we include an argument for the sake of completeness.

Proposition 4.4 (a). $M_{S}(X)$ is weak*-sequentially complete; (b) $\left(M_{S}(X), \tau(\xi)\right)$ is a complete locally convex space whose dual is $C^{*}(X)$.

Proof. (a) It is known [18] that $M_{\sigma}(X)$ is weak*-sequentially complete, hence we need only show that $M_{S}(X)$ is weak*-sequentially closed in $M_{\sigma}(X)$. But this is an immediate consequence of characterization (2) of 4.1 and [18, p. 183].

(b) The $\sigma\left(C^{*}, M_{S}\right)$-closed convex circled hull of any member of $\xi$ is in $\xi$, and consequently is $\sigma\left(C^{*}, M_{S}\right)$-compact since, from 4.2 and standard results, it is $\beta_{e}$-compact. Thus $\left(M_{S}(X), \tau(\xi)\right)^{\prime}=C^{*}(X)$ by the Mackey-Arens Theorem. Moreover, since $\mathscr{P} \leqq \sigma\left(C^{*}, M_{S}\right) \leqq \beta_{e}$, the other assertion follows from the characterization (3) of 4.1 and Grothendieck's Completeness Theorem.

If $L(X)$ denotes the linear span of the point masses in $M(X)$, then $L(X)$ is weak*-dense, hence $\tau(\xi)$-dense in $M_{S}(X)$. This shows that $\left(M_{S}(X), \tau(\xi)\right)$ is the completion of $(L(X), \tau(\xi))$.

5. Convergence in the space of measures. In this section we extend several known results on convergence of measures and answer a question posed on p. 393 of [13]:

(a) Do the weak*-topology and $\tau(\xi)$ coincide on

$$
\left\{\mu \in M_{S}^{+}(X): \mu(X)=1\right\} \quad(=Q X \text {, in the terminology of [13]) . }
$$

Dudley [5] showed that:

(b) If $\mu_{n} \stackrel{w^{*}}{\rightarrow} \mu$ in $M_{S}(X)$, then $\left(\mu_{n}\right)$ converges to $\mu$ uniformly on members of $\xi$ (i.e., in the topology $\tau(\xi)$ ).

Granirer [9] introduced the space of measures $D M_{\sigma}$ and showed (Theorems 1 and 2, and Remark 4, p. 15) that:

(c) Every member of $\xi$ is relatively $\sigma\left(C^{*}, D M_{\sigma}\right)$-compact and

(d) The weak*-topology and the topology $\tau(\xi)$ coincide on $D M_{\sigma}^{+}(X)$.

Our results are:

Proposition 5.1. $M_{\tau} \subset D M_{\sigma} \subset M_{S} \subset M_{\sigma}$.

Proposition 5.2. The finest locally convex topology on $C^{*}(X)$ for 
which members of $\xi$ are relatively compact is $\tau\left(C^{*}, M_{S}\right)$.

Proposition 5.3. The weak*-topology coincides with $\tau(\xi)$ on $M_{S}^{+}(X)$ and on relatively weak*-countably compact subsets of $M_{S}(X)$.

Proof of 5.1. We refer to [9] for the definitions of a $D$-space and the space of measures $D M_{\sigma}(X)$. It suffices to show that

$$
M_{\tau}^{+} \subset D M_{\sigma}^{+} \subset M_{S}^{+},
$$

since each of these cones generates the corresponding space of measures.

If $\mu \in M_{\tau}^{+}(X)$, then it is known $[11, p .338]$ that $\mu$ has an extension to a nonnegative countably-additive Borel measure $\nu$ satisfying (1) $\nu B=\inf \{\nu 0: 0$ open, $B \subset 0\}$ for any Borel set $B$; and (2) if $\left(F_{\alpha}\right)$ is a net of closed sets with $F_{\alpha} \downarrow \varnothing$, then $\nu F_{\alpha} \rightarrow 0$. Let

$$
C=\bigcap\{F: F \text { closed, } \nu F=\nu X\} ;
$$

then $\nu C=\nu X$. If $U$ is any nonempty open subset of $C$, then $U=$ $V \cap C$ where $V$ is open in $X$, and $\nu U=\nu V>0$. Thus $C$ satisfies the countable chain condition, hence is a $D$-space [9], and, for any Baire set $B$ with $C \subset B, \mu B=\nu B \geqq \nu C=\nu X=\mu X$. Thus

$$
\mu \in D M_{o}^{+}(X) \text {. }
$$

If $\mu \in D M_{\sigma}^{+}(X)$, let $S$ be a $D$-subspace of $X$ with $\mu^{*} S=\mu X$. Fix $d \in \mathscr{D}$, and let $P=\pi_{d}(S) \subset X_{d}$. If $e$ is a BCPM on $P$, then $e \circ\left(\pi_{d} \times \pi_{d}\right)$ is a BCPM on $S$, and it follows that $P$ is a $D$-space. If $B$ is a Baire set in $X_{d}$ and $P \subset B$, then $\pi_{d}^{-1} B$ is a Baire set in $X$ containing $S$, so that $\left(T_{d}^{*} \mu\right)(B)=\mu\left(\pi_{d}^{-1} B\right)=\mu X=T_{d}^{*} \mu\left(X_{d}\right)$. Thus $T_{d}^{*} \mu \in D M_{\sigma}^{+}\left(X_{d}\right)$, which coincides with $M_{\tau}^{+}\left(X_{d}\right)$ since $X_{d}$ is metric [9, p. 19]; hence $\mu \in M_{S}^{+}(X)$.

Granirer observes that $D M_{\sigma}(X)=M_{\sigma}(X)$ if and only if $X$ is a $D$-space; hence there are models for set theory in which

$$
D M_{\sigma}=M_{S}=M_{\sigma}
$$

(and $\beta_{e}=\beta_{1}$ ) for any space $X$. But unless it can be proved that real-valued measurable cardinals do not exist, $\beta_{e}$ and $\beta_{1}$ must be considered as distinct entities. Roughly speaking, a principal motivation for introducing $\beta_{e}$ is the hope of isolating the cardinality difficulties in the relation between $\beta_{e}$ and $\beta_{1}$, so that the equality of $\beta$ and $\beta_{e}$ can be investigated without reference to them.

Proof of 5.2. It is well-known that members of $\xi$ are relatively $\mathscr{P}$-compact. Thus $\beta_{e}$ is the finest locally convex topology with this 
property, from 4.2 , and $\beta_{e}=\tau\left(C^{*}, M_{S}\right)$, from 3.4 .

Proof of 5.3. If $E$ is any locally convex space, and $A$ is any equicontinuous subset of the dual $E^{\prime}$, then the weak*-topology and the topology of uniform convergence on totally bounded subsets of $E$ coincide when restricted to $A$. Let $E=\left(C^{*}(X), \beta_{e}\right)$, so that $E^{\prime}=$ $M_{S}(X)$. Since every relatively weak*-countably compact subset of $M_{S}(X)$ is $\beta_{e}$-equicontinuous (3.4), and every member of $\xi$ is relatively $\beta_{e}$-compact (5.2), hence $\beta_{e}$-totally bounded, the second half of the proposition is immediate.

Turning to the assertion about $M_{S}^{+}(X)$, let us first establish that, for $X$ a metric space, the weak*-topology and $\tau(\xi)$ coincide on $M_{\tau}^{+}(X)$ (this is a special case of Granirer's result (d), but an easy direct proof is possible). Now if $\beta_{e}$ and $M_{S}$ are replaced by $\beta$ and $M_{\tau}$ in the previous paragraph, it follows that $\sigma\left(M_{\tau}, C^{*}\right)$ and $\tau(\xi)$ coincide on weak*-compact subsets of $M_{\tau}^{+}$. But $\tau(\xi)$ is finer than $\sigma\left(M_{\tau}\right.$, $\left.C^{*}\right)$, and $\left(M_{*}^{+}, \sigma\left(M_{\tau}, C^{*}\right)\right)$ is metrizable, hence a $k$-space [6, p. 248]. It follows that $\tau(\xi)=\sigma\left(M_{\tau}, C^{*}\right)$ on $M_{\tau}^{+}(X)$.

Returning to the general case, suppose $\mu_{\alpha} \stackrel{w^{*}}{\rightarrow} \mu$ in $M_{S}^{+}(X)$, and let $E \in \xi(X)$. Constructing $d$ and $E_{d}$ as in 3.1(a), we have $T_{d}^{*} \mu_{\alpha} \stackrel{w^{*}}{\rightarrow} T_{d}^{*} \mu$ in $M_{\tau}^{+}\left(X_{d}\right)$, and so $\left(T_{d}^{*} \mu_{\alpha}\right)$ converges to $T_{d}^{*} \mu$ uniformly on $E_{d}$, from the previous paragraph. This is equivalent to saying that $\left(\mu_{\alpha}\right)$ converges to $\mu$ uniformly on $E$. Hence $\sigma\left(M_{S}, C^{*}\right)$ and $\tau(\xi)$ coincide on $M_{S}^{+}(X)$.

There is a natural embedding $j: X \rightarrow M(X)$, and a copy of the Stone-Cech compactification $\beta X$ can be constructed in $M^{+}(X)$ : it is simply the set of $\{0,1\}$-valued Baire measures in $M(X)$, with the relative weak*-topology. Moreover, if we identify $\beta X$ with this space, then $\beta X \cap M_{o}(X)=\nu X$, the Hewitt realcompactification of $X$, and $\beta X \cap M_{\tau}(X)=j(X)$.

Let $\mathscr{U}(\xi)$ be the uniformity on $M_{S}(X)$ of uniform convergence on members of $\xi$; then the uniform topology is of course $\tau(\xi)$. It is easy to show that the relative $\mathscr{C}(\xi)$ uniformity on $j(X)$ coincides with the finest compatible uniformity on $X$, often called the fine uniformity. The completion of $X$ with the fine uniformity is called the universal completion of $X$ and denoted $\theta X$; in [1] it is shown that $\theta X$ can be realized (topologically) as a subspace of $\beta X$. Now we give an alternate characterization of $\theta X$.

Corollary 5.4. The space $\beta X \cap M_{S}(X)$ of $\{0,1\}$-valued Baire measures in $M_{S}(X)$, with the relative weak*-topology and $\mathscr{C}(\xi)$ uniformity, is homeomorphic and uniformly isomorphic to $\theta X$. 
Proof. Since $\beta X$ is weak *-closed in $M(X), \beta X \cap M_{S}(X)$ is weak*closed, hence $\tau(\xi)$-closed, in $M_{S}(X)$, and therefore is $\mathscr{U}(\xi)$-complete, from $4.4(\mathrm{~b})$. Also $\sigma\left(M_{S}, C^{*}\right)$ and $\tau(\xi)$ coincide on $M_{S}^{+}(X)$ (5.3), so that $j(X)$ is $\tau(\xi)$-dense in $\beta X \cap M_{S}(X)$. The result now follows immediately.

Ideas similar to those expressed by 5.4 and preceding remarks (with the exception of the consequences of 5.3) are considered, at least implicitly, in [13].

6. Totally bounded sets and convergent sequences. If $X$ is locally compact, then $\beta$-totally bounded sets are relatively $\beta$-compact, and coincide with the members of $\xi$. Since $\mathscr{P} \leqq \beta_{0} \leqq \beta \leqq \beta_{e}$, we have seen that members of $\xi$ are relatively $\beta_{0}$ and $\beta$-compact in general, even relatively $\beta_{1}$-compact if $X$ is a $D$-space. Unfortunately, the converse fails.

EXAMPLE 6.1. A space $X$ with $\beta_{0}=\beta=\beta_{e}=\beta_{1}$ such that $C^{*}(X)$ contains a $\beta_{1}$-null sequence which is not equicontinuous. Let $N$ denote the positive integers with the discrete topology, let $p$ be a point of $\beta N \backslash N$, and let $X=N \cup\{p\}$. As pointed out by Varadarajan [18, p. 227], $X$ satisfies Prohorov's Theorem: every weak*-compact subset of $M_{t}^{+}(X)$ is uniformly tight. Since $X$ is $\sigma$-compact, $M_{o}(X)=$ $M_{t}(X)$, and so $X$ is $\beta$-simple, in the terminology of [14]: $\beta_{0}=\beta=\beta_{1}$. Now compact subsets of $X$ are finite, so if $f_{n}$ is the characteristic function of $\{n\}$, then $\left(f_{n}\right)$ is $\beta_{0}$-convergent to 0 , but obviously not equicontinuous at $p$.

A space $X$ is a $k_{R}$-space if the continuity of a real-valued function on $X$ is implied by its continuity when restricted to compact subsets of $X$. Every $k$-space is a $k_{R}$-space, but the converse is not true. It is konwn [17] that when $X$ is a $k_{R}$-space, $\beta_{0}$ is complete and $\beta$ and $\beta_{1}$ are sequentially complete.

Proposition 6.2. If $X$ is a $k_{R}$-space, then the following classes of subsets of $C^{*}(X)$ coincide: (1) $\xi$; (2) relatively $\beta$-compact; (3) $\beta$ totally bounded; (4) relatively $\beta_{0}$-compact; (5) $\beta_{0}$-totally bounded.

Proof. We have already noted that $(1) \rightarrow(2)$, and clearly $(2) \rightarrow$ $(3) \rightarrow(5) \rightarrow(4)$, the last implication following from $\beta_{0}$-completeness. Any relatively $\beta_{0}$-compact set is uniformly bounded and relatively compact in the compact-open topology, hence the implication (4) $\rightarrow$ (1) is an immediate consequence of Ascoli's Theorem [10, p. 234]. 
Sentilles has introduced $\beta_{0}^{+}$and $\beta^{+}$, the finest locally convex topologies on $C^{*}(X)$ with the same convergent sequences as $\beta_{0}$ and $\beta$, respectively, and inquired as to the relationship between $\beta_{0}^{+}$and $\beta$.

CoRollary 6.3. If $X$ is a $k_{R^{-}}$space, then $\beta_{0}$ and $\beta$ have the same convergent sequences, and $\beta \leqq \beta^{+}=\beta_{0}^{+} \leqq \beta_{1}$. If also $X$ is a $D$ space, then $\beta_{0}, \beta$, and $\beta_{1}$ have the same convergent sequences.

Proof. Obviously $\beta \leqq \beta^{+}$, and $\beta^{+} \leqq \beta_{1}^{+}=\beta_{1}$ was established in [17]. Moreover, $\beta_{0}^{+}=\beta^{+}=\beta_{e}^{+} \geqq \beta_{e}$ follows from 6.2. Thus if $X$ is a $D$-space, $\beta_{e}=\beta_{1}$, and the second assertion follows.

7. An open question. Theorems 3.4 and 3.7 show that the measure-theoretic condition $M_{S}^{+}=M_{\tau}^{+}$is sufficient for $\left(C^{*}(X), \beta\right)$ to be a strong Mackey space; this unifies the classes of paracompact spaces and measure-compact spaces (spaces for which $M_{\sigma}=M_{\tau}$ ). As a consequence of 5.4, a space $X$ for which $M_{S}^{+}=M_{\tau}^{+}$admits a complete uniform structure (this is the analogue of the known result that a measure-compact space is realcompact). This raises the question of a possible converse: if $\left(C^{*}(X), \beta\right)$ is a Mackey space, must $X$ admit a complete uniform structure?

Added in proof. Since submitting this article the author has learned that some of his results on the space of separable measures have also been obtained, independently and using different techniques, by Michel Rôme (C. R. Acad. Sci. Paris 274 (1972), 1631-1634, 18171820) and Richard Haydon ("On compactness in spaces of measures and measure-compact spaces," preprint).

\section{REFERENCES}

1. H. Buchwalter and R. Pupier, Caractérisation topologique de la complétion universelle d'un espace topologique completement régulier, C. R. Acad. Sc. Paris, 268 (1969), 1534-1536.

2. R. C. Buck, Bounded continuous functions on a locally compact space, Michigan Math. J., 5 (1958), 95-104.

3. H. S. Collins and J. R. Dorroh, Remarks on certain function spaces, Math. Ann., 176 (1968), 157-168.

4. J. B. Conway, The strict topology and compactness in the space of measures, Trans. Amer. Math. Soc., 126 (1967), 474-486.

5. R. M. Dudley, Convergence of Baire measures, Studia Math., 27 (1966), 251-268.

6. J. Dugundji, Topology, Allyn and Bacon, Boston, 1966.

7. D. H. Fremlin, D. J. H. Garling, and R. G. Haydon, Bounded measures on topological spaces, Proc. London Math. Soc., (3) 25 (1972), 115-136.

8. D. J. H. Garling, A generalized form of inductive-limit topology for vector spaces, Proc. London Math. Soc., 14 (1964), 1-28. 
9. E. Granirer, On Baire measures on D-topological spaces, Fund. Math., 60 (1967), $1-22$.

10. J. L. Kelley, General Topology, Van Nostrand, 1955.

11. R. B. Kirk, Locally compact, B-compact spaces, Nederl. Akad. Wetensch. Proc. Ser., A 72 (1969), 333-344.

12. L. LeCam, Convergence in Distribution of Stochastic Processes, Univ. California Publ. Statistics, 1958.

13. C. Leger and P. Soury, Le convexe topologique des probabilités sur un espace topologique, J. Math. Pures et Appl. 50 (1971), 363-425.

14. S. E. Mosiman and R. F. Wheeler, The strict topology in a completely regular setting: relations to topological measure theory, Canad. J. Math., 24 (1972), 873-890. 15. J. D. Pryce, $A$ device of $R$. J. Whitley's applied to pointwise compactness in spaces of continuous functions, Proc. London Math. Soc., 23 (1971), 532-546.

16. A. P. Robertson and W. J. Robertson, Topological Vector Spaces, Cambridge U. Press, 1966.

17. F. D. Sentilles, Bounded continuous functions on a completely regular space, Trans. Amer. Math. Soc., 168 (1972), 311-336.

18. V. S. Varadarajan, Measures on topological spases, Amer. Math. Soc. Translations, (2) 48 (1965), 161-228.

Received April 12, 1972.

NORTHERN ILLINOIS UNIVERSITY 


\section{PACIFIC JOURNAL OF MATHEMATICS}

\section{EDITORS}

D. Gilbarg and J. Milgram

Stanford University

Stanford, California 94305

R. A. Beaumont

University of Washington

Seattle, Washington 98105
J. DUGUNDJI* Department of Mathematics

University of Southern California Los Angeles, California 90007

RICHARD ARENS

University of California

Los Angeles, California 90024

\section{ASSOCIATE EDITORS}

E. F. BeCKenbach

B. H. NeUMaNN

F. WOLF

K. Yoshida

\section{SUPPORTING INSTITUTIONS}

UNIVERSITY OF BRITISH COLUMBIA

UNIVERSITY OF SOUTHERN CALIFORNIA

CALIFORNIA INSTITUTE OF TECHNOLOGY

UNIVERSITY OF CALIFORNIA

MONTANA STATE UNIVERSITY

STANFORD UNIVERSITY

UNIVERSITY OF TOKYO

UNIVERSITY OF NEVADA

UNIVERSITY OF UTAH

NEW MEXICO STATE UNIVERSITY

WASHINGTON STATE UNIVERSITY

OREGON STATE UNIVERSITY

UNIVERSITY OF OREGON

OSAKA UNIVERSITY

UNIVERSITY OF WASHINGTON

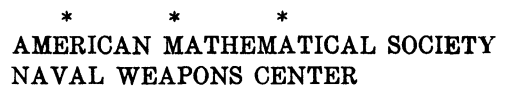

* C. DePrima will replace J. Dugundji until August 1974. 


\section{Pacific Journal of Mathematics}

\section{Vol. 47, No. $1 \quad$ January, 1973}

K. Adachi, Masuo Suzuki and M. Yoshida, Continuation of holomorphic

mappings, with values in a complex Lie group ....................

Michael Aschbacher, A characterization of the unitary and symplectic groups

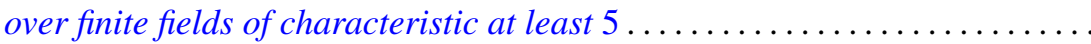

Larry Eugene Bobisud and James Calvert, Energy bounds and virial theorems for abstract wave equations....................................

Christer Borell, A note on an inequality for rearrangements ................

Peter Southcott Bullen and S. N. Mukhopadhyay, Peano derivatives and general

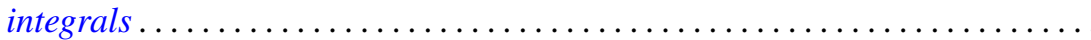

Wendell Dan Curtis, Yu-Lee Lee and Forrest Miller, A class of infinite dimensional subgroups of $\operatorname{Diff}^{r}(X)$ which are Banach Lie groups .........

Paul C. Eklof, The structure of ultraproducts of abelian groups ...............

William Alan Feldman, Axioms of countability and the algebra $C(X) \ldots \ldots \ldots$

Jack Tilden Goodykoontz, Jr., Aposyndetic properties of hyperspaces...........

George Grätzer and J. Płonka, On the number of polynomials of an idempotent algebra. II ...........................................

Alan Trinler Huckleberry, The weak envelope of holomorphy for algebras of

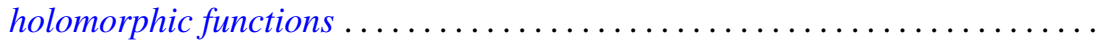

John Joseph Hutchinson and Julius Martin Zelmanowitz, Subdirect sum decompositions of endomorphism rings . . . . . . . . . . . . . . . .

Gary Douglas Jones, An asymptotic property of solutions of

$y^{\prime \prime \prime}+p y^{\prime}+q y=0$.

Howard E. Lacey, On the classification of Lindenstrauss spaces .

Charles Dwight Lahr, Approximate identities for convolution measure algebras.

George William Luna, Subdifferentials of convex functions on Banach

spaces.

Nelson Groh Markley, Locally circular minimal sets. .

Robert Wilmer Miller, Endomorphism rings of finitely generated projective modules

Donald Steven Passman, On the semisimplicity of group rings of linear

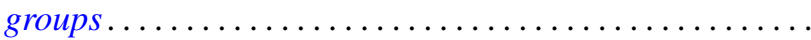

Bennie Jake Pearson, Dendritic compactifications of certain dendritic spaces.

Ryōtarō Satō, Abel-ergodic theorems for subsequences ...... .

Henry S. Sharp, Jr., Locally complete graphs. . .

Harris Samuel Shultz, A very weak topology for the Mikusinski field of

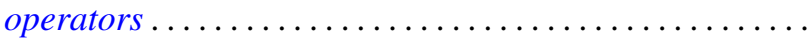

Elena Stroescu, Isometric dilations of contractions on Banach spaces ...

Charles W. Trigg, Versum sequences in the binary system ... . .

William L. Voxman, On the countable union of cellular decompositions of n-manifolds 